\title{
Safety assessment of essential medicines for elderly people: a bibliographic survey
}

\author{
Fabiana Rossi Varallo, Fernanda Mariana de Oliveira, Patrícia de Carvalho Mastroianni*
}

${ }^{I}$ School of Pharmaceutical Sciences, University of State of São Paulo “Júlio de Mesquita Filho”, Araraquara, SP, Brazil,

\begin{abstract}
Certain medicines are considered potentially inappropriate (PIM) for elderly people as they increase the risk of adverse drug events (ADE) and because safer alternative therapies are available on the market. In this context, in order to identify the instruments that assess the quality of medical prescriptions for elderly and to determine which drugs are considered PIM, a bibliographic survey was conducted in PUBMED, LILACS and PAHO databases, in February and March/2010. The search strategy included the use of health descriptors and a manual search in the references cited by selected papers. During the period of data collection, 15 instruments were identified. In 2012, with the publication of the update of Beers criteria, this instrument was included in the study. We identified 163 PIM of 25 therapeutic classes, of which 125 (76.7\%) are marketed in Brazil. Of these, 31 (24.8\%) are essential medicines (RENAME 2012), of which 13 have safer therapeutic equivalents and 19 (15.2\%) are over-the-counter drugs. Data suggest the need for inclusion of safer alternatives for the elderly in the national list of essential medicines and the pharmaceutical care for early detection of $\mathrm{ADE}$ in this age group, in order to contribute to the safe use of medicines.
\end{abstract}

Uniterms: Aged/medicines prescription. Medication/errors. Medicines/inappropriate prescribing. Medicines/adverse effects/control. Medicines/safety use. Medication/risk assessment/control. Pharmaceutical care.

\begin{abstract}
Determinados medicamentos são considerados potencialmente inapropriados (MPI) para idosos, por aumentarem o risco de ocorrência de eventos adversos a medicamentos (EAM) e por existirem alternativas terapêuticas mais seguras. Neste contexto, com o intuito de identificar os instrumentos que avaliam a qualidade das prescrições médicas para idosos e verificar quais medicamentos são considerados MPI, levantamento bibliográfico foi realizado nas bases de dados PUBMED, LILACS e PAHO em fevereiro e março de 2010. Para a seleção dos manuscritos utilizaram-se descritores em saúde e busca manual nas referências bibliográficas dos artigos identificados. No período da coleta de dados, foram identificados 15 instrumentos. Em 2012, com a publicação da atualização da lista de Beers, este instrumento foi incluído no estudo. Foram identificados 163 MPI de 25 classes terapêuticas, dos quais 125 (76,7\%) são comercializados no Brasil. Destes, 31 (24,8\%) são medicamentos essenciais (RENAME 2012), sendo que para 13 deles há equivalentes terapêuticos mais seguros e 19 (15,2\%) são medicamentos isentos de prescrição. Os dados sugerem a necessidade de inclusão de medicamentos mais seguros para idosos na lista nacional de medicamentos essenciais e do monitoramento farmacoterapêutico para a detecção precoce de EAM nesta faixa etária para contribuir com o uso seguro de medicamentos.
\end{abstract}

Unitermos: Idoso/prescrição de medicamentos. Medicação/erros. Medicamentos/prescrição inadequada. Medicamentos/efeitos adversos/controle. Medicamentos/uso seguro. Medicação de risco/controle. Monitoramento farmacoterapêutico.

Correspondence: P. C. Mastroianni. Faculdade de Ciências Farmacêuticas, Universidade Estadual Paulista "Júlio de Mesquita Filho". Rodovia Araraquara Jaú, km 1, 14801-902 - Araraquara - SP, Brasil. E-mail: pmastro@fcfar.unesp.br 


\section{INTRODUCTION}

Population aging is a worldwide phenomenon. However, this process is occurring evenly over all countries. In $2050,80 \%$ of all elderly people will be distributed among the developing nations (WHO, 2004). Thus, the United Nations Initiative (UNS, 1999) suggested that all countries should prepare their health care systems, as well as economic planning and social services in order to meet the needs of the elderly population.

In particular, the development of public health policies have to be developed for the elderly, in order to provide them with quality health care. In Brazil, the main laws are: the National Health Policy for the Elderly (Brasil, 1994), which aims "to promote healthy aging, with maintenance and improvement of their health condition, [...] functional capacity, and the prevention of disease and restoration of the health"; the Statute of Elderly People (Brasil, 2003), which ensures the comprehensive health care for the elderly, provided by the National Health Service (SUS), ensuring universal and equal access in an integrated and continuous set of actions and services for the prevention, promotion, protection and restoration of health.

Brazil is in a process of demographic transition, in which a marked aging of the population is evident. According to IBGE (2013), 33.7\% of population will be more than 60 years old by 2060 . Consequently, there is also an epidemiological transition, with important changes in the morbidity and mortality rates (IBGE, 2009), since the country will have changed the mortality profile until recently typical of young citizens, for a profile characteristic of senescence, with more complex and costly diseases (Gordilho et al., 2000).

Elderly patients who have complex medical problems and use polypharmacy (taking five or more drugs) are particularly more likely to be affected by medication errors (Fiolavá, Onder, 2009), such as the prescription of potentially inappropriate medications (PIM), and to be hospitalized (De Paula et al., 2012). Moreover, the inherent physiological changes of the aging process may influence the pharmacokinetics and pharmacodynamics of a drug, resulting in greater sensitivity to both its therapeutic and adverse effects (Corsonello et al., 2010).

PIM are those medications or classes of medication that should be avoided in patients aged $\geq 65$ years, since they have no clear evidence-based efficacy, are not costeffective (Beers et al., 1991) and on pose unnecessary risk to the health of the elderly, in that the risks of using those drugs exceed the benefits and safer alternatives are available (Fick et al., 2003; Gallagher et al., 2008).
One way to assess the quality of drug prescriptions for elderly patients is to use instruments that incorporate explicit (Bongue et al., 2009) and implicit indicators. The former list the drugs considered inappropriate for this age group and the latter consider the pathophysiology of the patient and the use of concomitant medications. However, these instruments are only tools that can guide drug prescription (Smet et al., 2007; Spinewine et al., 2007; Castelino et al., 2009) and must not replace clinical and pharmacotherapeutic assessment for the selection of the most appropriate drug in order to obtain the best results (Castelino et al., 2009).

Several criteria that evaluate the safety of pharmacotherapy for elderly people have been published, however no one criteria is established as a gold standard. Therefore, the aim of this study was to: 1) identify the instruments developed to assess the safety of pharmacotherapy for elderly people; 2) determine the drugs considered potentially inappropriate for this age group, according to the instruments identified, and 3) identify the PIM contained in the Brazilian list of essential drugs (RENAME), in order to propose safer therapeutic equivalents for elderly people.

\section{METHODS}

During February and March of 2010, a bibliographic survey was performed in Pubmed, LILACS and PAHO databases (available at http://regional.bvsalud.org/php/ index.php), in order to identify the original studies that developed or used instruments to analyze the quality of drug prescriptions for elderly people (aged $\geq 65$ years). The search strategy adopted the following scientific health descriptors: "drug prescription" AND "aged" OR "elderly people" AND "medication errors", as well as the key words: "screening tool" AND "inappropriate medications". Other relevant publications were localized by a manual search, consulting the references of selected articles.

Original studies published in journals indexed in the databases consulted, which were written in the English, Portuguese or Spanish languages and developed and/ or applied instruments that contained implicit or explicit indicators for quality analysis of drug prescription for elderly people were considered eligible for the present review. The exclusion criteria comprised: review manuscripts, editorials, letters, news, abstracts of conference proceedings and data from thesis and dissertations.

The articles were independently selected by two reviewers, using the techniques of floating reading and content analysis. The first allowed the pre-selection of primary studies whose titles and abstracts referred to the 
theme of the study. Each pre-selected study was assessed by the technique of content analysis, in order to verify that it matched the inclusion criteria previously established and to identify the drugs considered inappropriate for use in senescence.

The drugs classified as PIM by the instruments identified were analyzed, in order to determine whether they are present in the 16th WHO Model List of Essential Medicines (WHO, 2010) and in the List of Essential Medicines in Brazil (RENAME, 2010) (Brasil, 2010). For those included in the criteria of essential medicines (PIM present in WHO and RENAME lists), safer equivalent therapies were suggested, where possible, on the basis of articles consulted and the pharmacological characteristics of the drugs.

Since the implicit criteria require clinical evaluation of patient and of the drugs taken, the instruments using these indications do not list the PIM for the elderly population, since any drug could be considered inappropriate. For this reason, they were not considered for data tabulation.

\section{RESULTS}

By the search strategy proposed, 203 articles were identified. Of these, 156 satisfied the exclusion criteria and 47 had their content analyzed. After content analysis, 12 of these articles were excluded, since they did not refer to the instruments used to evaluate the safety of pharmacotherapy for elderly people. After this process, 34 articles were considered eligible for the study.

Fifteen instruments to assess the quality of drug prescriptions for elderly people were identified: the Beers criteria (1991) (Beers et al., 1991) and their updates in 1997 (Beers, M.H.; 1997), 2003 (Fick et al., 2003), the Medication Appropriateness Index (MAI) (Hanlon et al., 1992), the Lipton (Lipton et al., 1993) and McLeod (McLeod et al., 1997) criteria, the HEDIS method (Pugh et al., 2006), Norwegian quality indicators for prescribing (Brekke et al., 2008), Swedish quality indicators of prescription (Johnell, Fastbom, 2008), the Improving Prescribing in the Elderly Tool (Ipet) (Naugler et al., 2000), the French list of inappropriate drugs (Laroche et al., 2007), the Norwegian list of inappropriate drugs (Straand, Rokstad, 1999), the Screening Tool to Alert doctors to the Right Treatment (START) (Barry et al., 2007), Zhan criteria (Zhan et al., 2001), and the Screening Tool of Older Persons' Potentially Inappropriate Prescriptions (STOPP) (Gallagher, O'Mahony, 2008). In 2012, a new version of the Beers criteria (AGS, 2012) was published. Therefore, in order to update our findings, this instrument was included in our revision. Thus, we found 16 instruments.

Of these instruments, the MAI (Hanlon et al., 1992) and the Lipton criteria (Lipton et al., 1993) incorporate implicit indicators for the assessment of the quality of prescriptions for the elderly.

The instruments STOPP (Screening Tool of Older Persons' Prescriptions) and START (Screening Tool to Alert doctors to the Right Treatment) (Gallagher et al., 2008) list 8 therapeutic classes of potentially inappropriate medications for the elderly.

Eleven instruments (Beers et al., 1991; Beers, 1997; Straand, Rokstad, 1999; Naugler et al., 2000; Zhan et al., 2001; Fick et al., 2003; Pugh et al., 2006; Laroche et al., 2007; Brekke et al., 2008; Johnell, Fastbom, 2008; AGS, 2012) considered 163 drugs of 25 therapeutic classes PIM for elderly people, which should be avoided, irrespective of the diagnosis or conditions of the patient (Table I). According to WHO the Anatomical Therapeutic Chemical (ATC) Classification system, 33.1\% (54/163) of PIM act on the nervous system, $16.5 \%(27 / 163)$ on the cardiovascular system, $6.7 \%(11 / 163)$ on the respiratory system, $10.4 \%(17 / 163)$ on the musculoskeletal system, $8.6 \%(14 / 163)$ on the alimentary tract and metabolism, $3.7 \%(6 / 163)$ on genitourinary system and sex hormones, and $1.8 \%(3 / 163)$ on blood and hematopoietic organs, while $0.6 \%(1 / 163)$ are systemic hormones and $0.6 \%$ $(1 / 163)$ are anti-infective. Another 18 drugs found were classified as acting on 2 or more ATC therapeutic classes, while 14 drugs were not found in this classification.

The 2012 updating of Beers criteria (AGS, 2012) covers $72.4 \%$ of all PIM identified in the assessed studies, followed by the 2003 updating of Beers criteria (Fick et $a l ., 2003$ ) and by the list developed in France (Laroche et al., 2007) (Table II).

Finally, it was observed that 19 PIM are present in the 16th WHO Model List of Essential Medicines (WHO, 2010) and 20 (5.8\%) in the RENAME 2010 (Table III). Thus, these drugs are considered essential, according to the criteria of cost, safety, quality and effectiveness. Regarding the proposal of safer equivalent therapies, seven PIM classified as essential medicines in the Rename list have no safer equivalents (Table III). When we examined the PIM classified as essential drugs in the WHO list, in eight cases it was not possible to propose safer equivalent therapies for elderly people (Table III).

In 2012, there was an update of the RENAME list, which now includes 31 PIM (acetylsalicylic acid, cáscara sagrada, chlorpromazine, fluoxetine, metoclopramide, promethazine, clobazam, clonazepam, amiodarone, amitriptyline, clomipramine, propaphenone, propranolol, 
TABLE I - List of potentially inappropriate medications (PIM) for the elderly that should be avoided irrespective of diagnosis or clinical condition, according to the methods identified in PAHO (available in: http://regional.bvsalud.org/php/index.php), Lilacs and Pubmed databases, during the period between February and March $2010(\mathrm{n}=12)$

\begin{tabular}{|c|c|c|c|c|c|c|c|c|c|c|c|c|}
\hline Drugs / ATC Code & 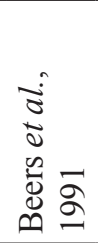 & 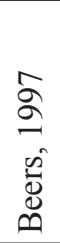 & 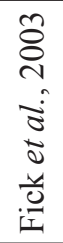 & 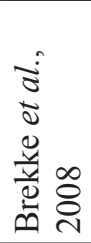 & 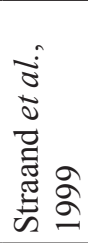 & 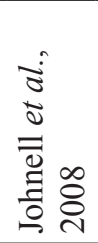 & 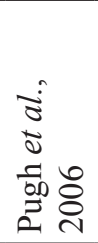 & 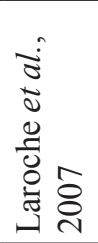 & 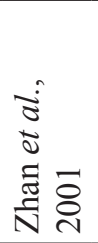 & 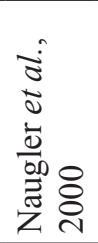 & 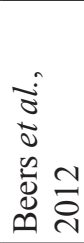 & 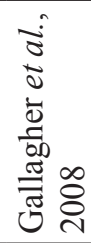 \\
\hline Ethacrynic acid (C03CC01) & & & $\mathrm{X}$ & & & & & & & & & \\
\hline Analgesics & & & & & & & & & & & & $\mathrm{X}$ \\
\hline Salicylic acid $(>325 \mathrm{mg} / \mathrm{d})(\mathrm{N} 02 \mathrm{BA} 01)$ & & & & & & & & & & & $\mathrm{X}$ & \\
\hline Diclofenac (M01AB05) & & & & & & & & & & & $\mathrm{X}$ & \\
\hline Diflunisal (N02BG) & & & & & & & & & & & $\mathrm{X}$ & \\
\hline Etodolac (M01AB08) & & & & & & & & & & & $\mathrm{X}$ & \\
\hline Fenoprofen (M01AE04) & & & & & & & & & & & $\mathrm{X}$ & \\
\hline Ibuprofen (M01AE01) & & & & & & & & & & & $\mathrm{X}$ & \\
\hline Indometacin (M01AB01) & $\mathrm{X}$ & $\mathrm{X}$ & $\mathrm{X}$ & & $\mathrm{X}$ & & & $\mathrm{X}$ & $\mathrm{X}$ & & $\mathrm{X}$ & \\
\hline Ketoprofen (M01AE03) & & & & & & & & & & & $\mathrm{X}$ & \\
\hline Ketorolac (M01AB15) & & & $\mathrm{X}$ & & & & $\mathrm{X}$ & & & & $\mathrm{X}$ & \\
\hline Mefenamic acid (M01AG01) & & & & & & & & & & & $\mathrm{X}$ & \\
\hline Meloxicam (M01AC06) & & & & & & & & & & & $\mathrm{X}$ & \\
\hline Nabumetone (M01AX01) & & & & & & & & & & & $\mathrm{X}$ & \\
\hline Naproxen (M01AE02) & & & $\mathrm{X}$ & & & & & & & & $\mathrm{X}$ & \\
\hline Oxaprozin (M01AE12) & & & $\mathrm{X}$ & & & & & & & & $\mathrm{X}$ & \\
\hline Phenylbutazone (M01AA01) & $\mathrm{X}$ & $\mathrm{X}$ & & & & & & $\mathrm{X}$ & & & & \\
\hline Piroxicam (M01AC01) & & & $\mathrm{X}$ & & & & & & & & $\mathrm{X}$ & \\
\hline Sulindac (M01AB02) & & & & & & & & & & & $\mathrm{X}$ & \\
\hline Tolmetin (M01AB03) & & & & & & & & & & & $\mathrm{X}$ & \\
\hline \multicolumn{13}{|l|}{ Antiparkinson agents } \\
\hline Benzatropine (N04AC01) & & & & & & & & & & & $\mathrm{X}$ & \\
\hline Trihexyphenidyl (N04AA01) & & & & & & & & & & & $\mathrm{X}$ & \\
\hline Antispasmodic & $\mathrm{X}$ & & & & & & & & & & & \\
\hline Belladonna alkaloids (A03BA) & & $\mathrm{X}$ & $\mathrm{X}$ & & & & $\mathrm{X}$ & & $\mathrm{X}$ & & $\mathrm{X}$ & \\
\hline Clidinium-chlordiazepoxide (A033CA02) & $\mathrm{X}$ & $\mathrm{X}$ & $\mathrm{X}$ & & & & & & & & $\mathrm{X}$ & \\
\hline Dicyclomine (A03AA07) & & $\mathrm{X}$ & $\mathrm{X}$ & & & & $\mathrm{X}$ & & $\mathrm{X}$ & & $\mathrm{X}$ & \\
\hline Hyoscyamine (A03BA03) & & $\mathrm{X}$ & $\mathrm{X}$ & & & & $\mathrm{X}$ & & $\mathrm{X}$ & & $\mathrm{X}$ & \\
\hline Propantheline (A03AB05) & & $\mathrm{X}$ & & & & & $\mathrm{X}$ & & $\mathrm{X}$ & & $\mathrm{X}$ & \\
\hline Oxybutynin (G04BD04) & & $\mathrm{X}$ & $\mathrm{X}$ & & & $\mathrm{X}$ & & $\mathrm{X}$ & $\mathrm{X}$ & & & \\
\hline Scopolamine (A04AD01) & & & & & & & & & & & $\mathrm{X}$ & \\
\hline \multicolumn{13}{|l|}{ Anti-emetics } \\
\hline Trimethobenzamide (none) & $\mathrm{X}$ & $\mathrm{X}$ & $\mathrm{X}$ & & & & $\mathrm{X}$ & & $\mathrm{X}$ & & & \\
\hline Amphetamines and anorexic oral anti-infectives & & & $\mathrm{X}$ & & & & $\mathrm{X}$ & & & & & \\
\hline Nitrofurantoin (J01XE01) & $\mathrm{X}$ & & $\mathrm{X}$ & & & & $\mathrm{X}$ & $\mathrm{X}$ & & & $\mathrm{X}$ & \\
\hline Anticholinergics & & & & & & $\mathrm{X}$ & & & & & $\mathrm{X}$ & \\
\hline Antidepressants & & & & & & $\mathrm{X}$ & & & & & & \\
\hline Amitriptyline (N06AA09) & $\mathrm{X}$ & $\mathrm{X}$ & $\mathrm{X}$ & $\mathrm{X}$ & $\mathrm{X}$ & & & $\mathrm{X}$ & $\mathrm{X}$ & $\mathrm{X}$ & $\mathrm{X}$ & \\
\hline Amoxapine (N06AA17) & & & & & & & & $\mathrm{X}$ & & & & \\
\hline Clomipramine (N06AA04) & & & & $\mathrm{X}$ & & & & $\mathrm{X}$ & & & $\mathrm{X}$ & \\
\hline Doxepin (N06AA12) & & $\mathrm{X}$ & $\mathrm{X}$ & $\mathrm{X}$ & & & & $\mathrm{X}$ & $\mathrm{X}$ & $\mathrm{X}$ & $\mathrm{X}$ & \\
\hline Fluoxetine (N06AB03) & & & $\mathrm{X}$ & & & & & & & & & \\
\hline Imipramine (N06AA02) & & & & & & & & $\mathrm{X}$ & & $\mathrm{X}$ & $\mathrm{X}$ & \\
\hline
\end{tabular}


TABLE I - List of potentially inappropriate medications (PIM) for the elderly that should be avoided irrespective of diagnosis or clinical condition, according to the methods identified in PAHO (available in: http://regional.bvsalud.org/php/index.php), Lilacs and Pubmed databases, during the period between February and March 2010 (n=12) (cont.)

\begin{tabular}{|c|c|c|c|c|c|c|c|c|c|c|c|c|}
\hline Drugs / ATC Code & 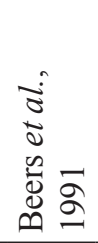 & $\begin{array}{l}\hat{\sigma} \\
\hat{a} \\
\hat{d} \\
\bar{d}\end{array}$ & 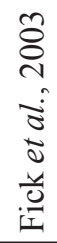 & 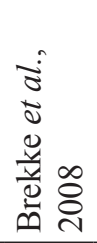 & 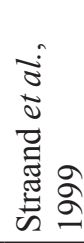 & 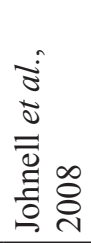 & 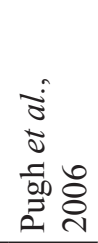 & 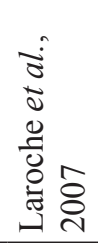 & 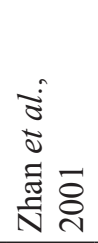 & 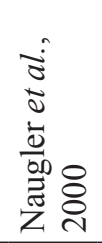 & 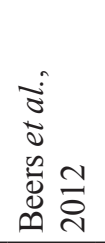 & 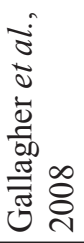 \\
\hline Maprotiline (N06AA21) & & & & & & & & $\mathrm{X}$ & & & & \\
\hline Trimipramine (N06AA06) & & & & $\mathrm{X}$ & & & & $\mathrm{X}$ & & & $\mathrm{X}$ & \\
\hline \multicolumn{13}{|l|}{ Oral antidiabetics } \\
\hline Carbutamide (A10BB06) & & & & & & & & $\mathrm{X}$ & & & & \\
\hline Chlorpropamide (A10BB02) & $\mathrm{X}$ & $\mathrm{X}$ & $\mathrm{X}$ & $\mathrm{X}$ & $\mathrm{X}$ & & $\mathrm{X}$ & & $\mathrm{X}$ & & $X$ & \\
\hline Glipizide (A10BB07) & & & & & & & & $\mathrm{X}$ & & & & \\
\hline Glibenclamide (A10BB01) & & & & & & & & & & & $\mathrm{X}$ & \\
\hline Antihistamines & & & & & & $\mathrm{X}$ & & & & & & \\
\hline Alimemazine (R06AD01) & & & & $\mathrm{X}$ & & & & $\mathrm{X}$ & & & & \\
\hline Brompheniramine (R06AB01) & & & & & & & & $\mathrm{X}$ & & & $\mathrm{X}$ & \\
\hline Carbinoxamine (R06AA08) & & & & & & & & $\mathrm{X}$ & & & $\mathrm{X}$ & \\
\hline Cimetidine (A02BA01) & $\mathrm{X}$ & & $\mathrm{X}$ & & & & & $\mathrm{X}$ & & & & \\
\hline Clemastine (R06AA04) & & & & & & & & & & & $\mathrm{X}$ & \\
\hline Cyproheptadine (R06AX02) & & $\mathrm{X}$ & $\mathrm{X}$ & & & & $\mathrm{X}$ & $\mathrm{X}$ & $\mathrm{X}$ & & $X$ & \\
\hline Chlorpheniramine (R06AB02) & & $\mathrm{X}$ & $\mathrm{X}$ & & & & $\mathrm{X}$ & & $\mathrm{X}$ & & $\mathrm{X}$ & \\
\hline Dexbrompheniramine(R06AB06) & & & & & & & & & & & $\mathrm{X}$ & \\
\hline Dexchlorpheniramine(R06AB02) & & $\mathrm{X}$ & $\mathrm{X}$ & $\mathrm{X}$ & & & $\mathrm{X}$ & $\mathrm{X}$ & & & $\mathrm{X}$ & \\
\hline Diphenhydramine (R06AA02) & & $\mathrm{X}$ & $\mathrm{X}$ & & & & $\mathrm{X}$ & & $\mathrm{X}$ & & $\mathrm{X}$ & \\
\hline Doxylamine (R06AA09) & & & & & & & & $\mathrm{X}$ & & & $\mathrm{X}$ & \\
\hline Hydroxyzine (N05BB01) & & $\mathrm{X}$ & $\mathrm{X}$ & $\mathrm{X}$ & & & $\mathrm{X}$ & $\mathrm{X}$ & $\mathrm{X}$ & & $\mathrm{X}$ & \\
\hline Promethazine (R06AD02) & & $\mathrm{X}$ & $\mathrm{X}$ & $\mathrm{X}$ & & & $\mathrm{X}$ & $\mathrm{X}$ & $\mathrm{X}$ & & $\mathrm{X}$ & \\
\hline Tripelennamine (R06AC04) & & $\mathrm{X}$ & $\mathrm{X}$ & & & & $\mathrm{X}$ & & & & & \\
\hline Triprolidine (R06AX07) & & & & & & & & & & & $X$ & \\
\hline \multicolumn{13}{|l|}{ Antiplatelet } \\
\hline Dipyridamole (B01AC07) & $\mathrm{X}$ & $\mathrm{X}$ & $\mathrm{X}$ & & $\mathrm{X}$ & & $\mathrm{X}$ & $\mathrm{X}$ & $\mathrm{X}$ & & $\mathrm{X}$ & \\
\hline Ticlopidine (B01AC05) & & $\mathrm{X}$ & $\mathrm{X}$ & & & & & $\mathrm{X}$ & $\mathrm{X}$ & & $X$ & \\
\hline Antipsychotics & & & & & & $\mathrm{X}$ & & & & & & \\
\hline Cyamemazine (N05AA06) & & & & & & & & $\mathrm{X}$ & & & & \\
\hline Chlorpromazine (N05AA01) & & & & $\mathrm{X}$ & & & & $\mathrm{X}$ & & & & \\
\hline Chlorprothixene (N05AF03) & & & & $\mathrm{X}$ & & & & & & & & \\
\hline Fluphenazine (N05AB02) & & & & & & & & $\mathrm{X}$ & & & & \\
\hline Haloperidol (N05AD01) & $\mathrm{X}$ & & & $\mathrm{X}$ & & & & $\mathrm{X}$ & & & & \\
\hline Levomepromazine (N05AA02) & & & & $\mathrm{X}$ & & & & $\mathrm{X}$ & & & & \\
\hline Mesoridazine (N05AC03) & & & $\mathrm{X}$ & & & & $\mathrm{X}$ & & & & $\mathrm{X}$ & \\
\hline Perphenazine (N05AB03) & & & & & & & & $X$ & & & & \\
\hline Pipotiazine (N05AC04) & & & & & & & & $\mathrm{X}$ & & & & \\
\hline Prochlorperazine (N05AB04) & & & & $\mathrm{X}$ & & & & & & & & \\
\hline Pericyazine (N05AC01) & & & & & & & & $X$ & & & & \\
\hline Thioridazine (N05AC02) & $\mathrm{X}$ & & $\mathrm{X}$ & & & & $\mathrm{X}$ & & & & $X$ & \\
\hline Cardiovascular & & & & & & & & & & & & $\mathrm{X}$ \\
\hline Amiodarone (C01BD01) & & & $\mathrm{X}$ & & & & & & & & $\mathrm{X}$ & \\
\hline Clonidine (C02AC01) & & & $\mathrm{X}$ & & & & & $\mathrm{X}$ & & & $\mathrm{X}$ & \\
\hline Digoxin (C01AA05) & & $\mathrm{X}$ & $\mathrm{X}$ & & & & & $\mathrm{X}$ & & & $\mathrm{X}$ & \\
\hline
\end{tabular}


TABLE I - List of potentially inappropriate medications (PIM) for the elderly that should be avoided irrespective of diagnosis or clinical condition, according to the methods identified in PAHO (available in: http://regional.bvsalud.org/php/index.php), Lilacs and Pubmed databases, during the period between February and March 2010 ( $\mathrm{n}=12)$ (cont.)

\section{Drugs / ATC Code}

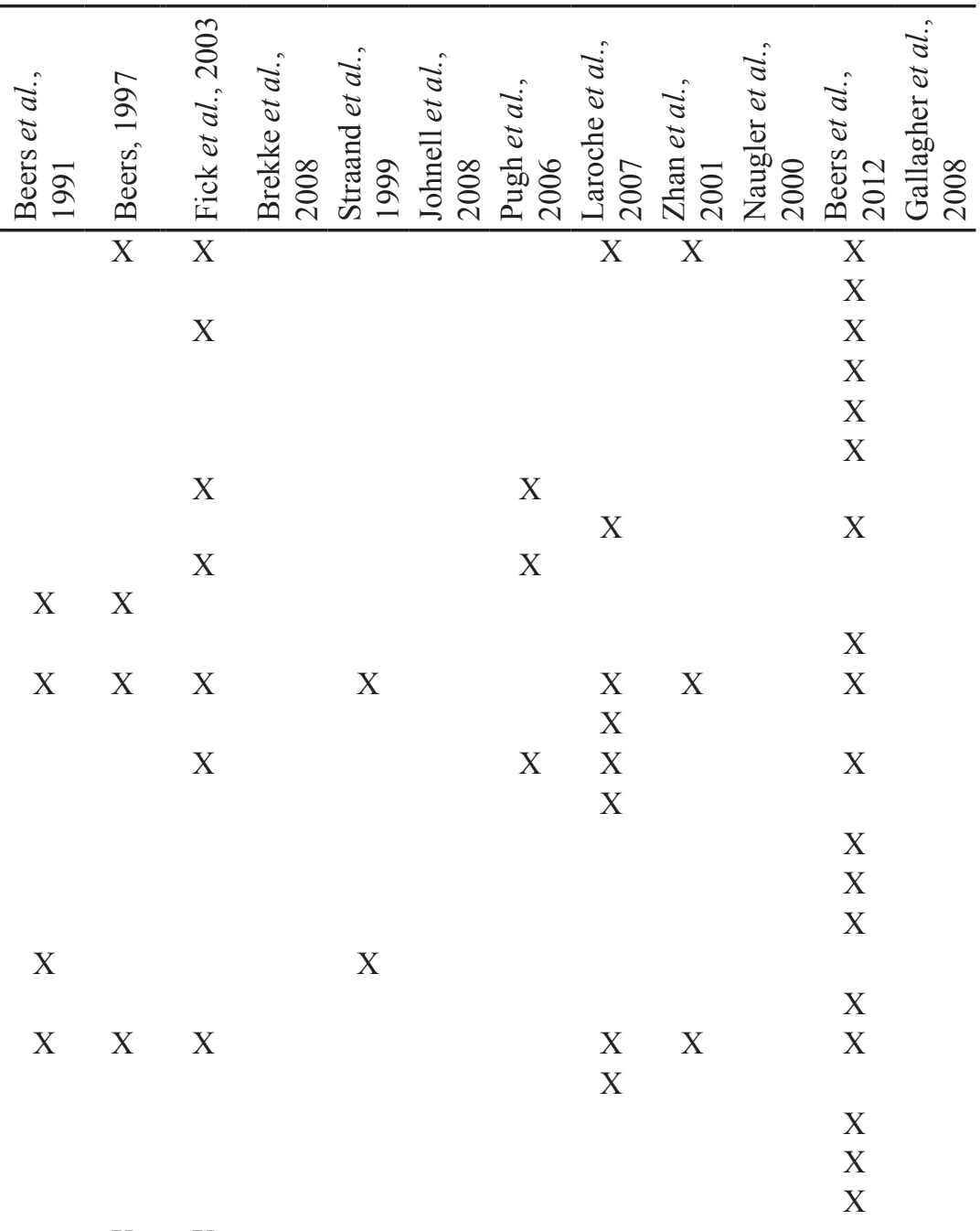

Ergot derivatives (C04AE)

\section{Descongestants}

Phenylephrine (R01AA04)

Pseudoephedrine (R01BA02)

Oxymetazoline (R01AA05)

\section{Hypnoanalgesics}

Meperidine (N02AB02)

Pentazocine (N02AD01)

Pethidine (N02AB02)

Propoxyphene (N02AC04)

Hypnotics/sedatives

\section{Barbiturates}

Amobarbital (N05CA02)

X X

$X$
$X$
$X$

Butabarbital (none)

Butalbital (none)

Mephobarbital (N03AA01)

Pentobarbital (N05CA01)

Phenobarbital (N03AA02)

$\begin{array}{ccccccccc} & \mathrm{X} & \mathrm{X} & & & & \mathrm{X} & \mathrm{X} & \mathrm{X} \\ \mathrm{X} & \mathrm{X} & \mathrm{X} & & \mathrm{X} & & \mathrm{X} & & \mathrm{X} \\ & & & \mathrm{X} & & & & & \\ \mathrm{X} & \mathrm{X} & \mathrm{X} & \mathrm{X} & \mathrm{X} & & \mathrm{X} & \mathrm{X} & \\ & & & & & \mathrm{X} & & & \\ & & & & & & \mathrm{X} & \mathrm{X} & \end{array}$

$\mathrm{X}$

$\mathrm{X}$

$\mathrm{X}$

$\mathrm{X}$

$\mathrm{X}$ 
TABLE I - List of potentially inappropriate medications (PIM) for the elderly that should be avoided irrespective of diagnosis or clinical condition, according to the methods identified in PAHO (available in: http://regional.bvsalud.org/php/index.php), Lilacs and Pubmed databases, during the period between February and March 2010 (n=12) (cont.)

\begin{tabular}{|c|c|c|c|c|c|c|c|c|c|c|c|c|}
\hline Drugs / ATC Code & 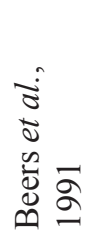 & 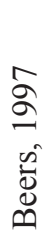 & 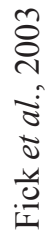 & 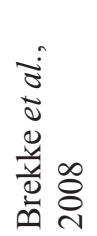 & 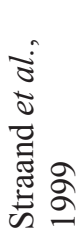 & 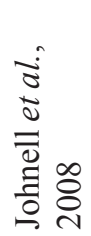 & 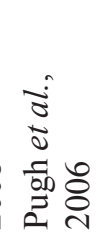 & 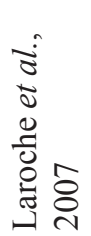 & 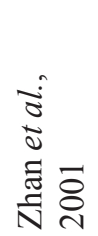 & 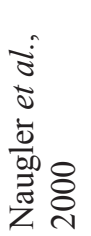 & 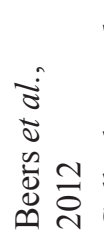 & 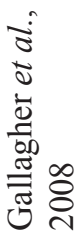 \\
\hline Secobarbital (N05CA06) & $\mathrm{X}$ & $\mathrm{X}$ & $\mathrm{X}$ & & & & & & & & $\mathrm{X}$ & \\
\hline \multicolumn{13}{|l|}{ Benzodiazepines } \\
\hline Long-term & & & & & & $\mathrm{X}$ & & & & $\mathrm{X}$ & & \\
\hline Bromazepam (N05BA08) & & & & & & & & $\mathrm{X}$ & & & & \\
\hline Chlordiazepoxide (N05BA02) & $\mathrm{X}$ & $\mathrm{X}$ & $\mathrm{X}$ & & & & $\mathrm{X}$ & $\mathrm{X}$ & $\mathrm{X}$ & & $\mathrm{X}$ & \\
\hline Clobazam (N05BA09) & & & & & & & & $\mathrm{X}$ & & & & \\
\hline Clonazepam (N03AE01) & & & & & & & & & & & $\mathrm{X}$ & \\
\hline Clorazepate (N05BA05) & & & $\mathrm{X}$ & & & & $\mathrm{X}$ & $\mathrm{X}$ & & & $\mathrm{X}$ & \\
\hline Diazepam (N05BA01) & $\mathrm{X}$ & $\mathrm{X}$ & $\mathrm{X}$ & & & $\mathrm{X}$ & $\mathrm{X}$ & & $\mathrm{X}$ & & $\mathrm{X}$ & \\
\hline Flurazepam (N05CD01) & $\mathrm{X}$ & $\mathrm{X}$ & $\mathrm{X}$ & & & & $\mathrm{X}$ & & & & $\mathrm{X}$ & \\
\hline Flunitrazepam (N05CD03) & & & & $\mathrm{X}$ & & $\mathrm{X}$ & & & & & & \\
\hline Halazepam (N05BA13) & & & $\mathrm{X}$ & & & & $\mathrm{X}$ & & & & & \\
\hline Loflazepate (N05BA18) & & & & & & & & $\mathrm{X}$ & & & & \\
\hline Nitrazepam (N05CD02) & & & & $\mathrm{X}$ & & $\mathrm{X}$ & & $\mathrm{X}$ & & & & \\
\hline Nordazepam (N05BA16) & & & & & & & & $\mathrm{X}$ & & & & \\
\hline Quazepam (N05CD10) & & & $\mathrm{X}$ & & & & $\mathrm{X}$ & & & & $\mathrm{X}$ & \\
\hline Prazepam (N05BA11) & & & & & & & & $\mathrm{X}$ & & & & \\
\hline Tetrazepam (M03BX07) & & & & & & & & $\mathrm{X}$ & & & & \\
\hline \multicolumn{13}{|l|}{ Short term } \\
\hline Alprazolam (N05BA12) & $\mathrm{X}$ & $\mathrm{X}$ & $\mathrm{X}$ & & & & & & & & $\mathrm{X}$ & \\
\hline Estazolam (N05CD04) & & & & & & & & $\mathrm{X}$ & & & $\mathrm{X}$ & \\
\hline Eszopiclone (N05CF04) & & & & & & & & & & & $\mathrm{X}$ & \\
\hline Lorazepam (N05BA06) & & $\mathrm{X}$ & $\mathrm{X}$ & & & & & & & & $\mathrm{X}$ & \\
\hline Oxazepam (N05BA04) & $\mathrm{X}$ & $\mathrm{X}$ & $\mathrm{X}$ & & & & & & & & $\mathrm{X}$ & \\
\hline Temazepam (N05CD07) & & $\mathrm{X}$ & $\mathrm{X}$ & & & & & & & & $\mathrm{X}$ & \\
\hline Triazolam (N05CD05) & $\mathrm{X}$ & $\mathrm{X}$ & $\mathrm{X}$ & & & & & & & & $\mathrm{X}$ & \\
\hline Zaleplon (N05CF03) & & & & & & & & & & & $\mathrm{X}$ & \\
\hline Zolpidem (N05CF02) & & $\mathrm{X}$ & & & & & & & & & $\mathrm{X}$ & \\
\hline Meprobamate (N05BC01) & $\mathrm{X}$ & $\mathrm{X}$ & $\mathrm{X}$ & & & & $\mathrm{X}$ & & & & $\mathrm{X}$ & \\
\hline \multirow{2}{*}{\multicolumn{13}{|c|}{$\begin{array}{l}\text { Endocrine } \\
\text { Androgens (G03B) }\end{array}$}} \\
\hline & & & & & & & & & & & & \\
\hline Methyltestosterone (G03BA02) & & & $\mathrm{X}$ & & & & $\mathrm{X}$ & & & & $\mathrm{X}$ & \\
\hline Testosterone (G03BA03) & & & & & & & & & & & $\mathrm{X}$ & \\
\hline Desiccated thyroid (H03) & & & $\mathrm{X}$ & & & & $\mathrm{X}$ & & & & $\mathrm{X}$ & \\
\hline Estrogen (G03C) & & & $\mathrm{X}$ & & & & $\mathrm{X}$ & & & & $\mathrm{X}$ & \\
\hline Growth hormone (L03) & & & & & & & & & & & $\mathrm{X}$ & \\
\hline Insulin, sliding scale (A10AB) & & & & & & & & & & & $\mathrm{X}$ & \\
\hline Megestrol (G03AC05) & & & & & & & & & & & $\mathrm{X}$ & \\
\hline Gastrointestinal/laxatives & & & & & & & & & & & & $\mathrm{X}$ \\
\hline Bisacodyl (A06AB02) & & & $\mathrm{X}$ & & & & & $\mathrm{X}$ & & & & \\
\hline Cáscara Sagrada (A06AB07) & & & $\mathrm{X}$ & & & & & $\mathrm{X}$ & & & & \\
\hline Metoclopramide (A03FA01) & & & & & & & & & & & $\mathrm{X}$ & \\
\hline Mineral oil (A06AA) & & & $\mathrm{X}$ & & & & & $\mathrm{X}$ & & & $\mathrm{X}$ & \\
\hline
\end{tabular}


TABLE I - List of potentially inappropriate medications (PIM) for the elderly that should be avoided irrespective of diagnosis or clinical condition, according to the methods identified in PAHO (available in: http://regional.bvsalud.org/php/index.php), Lilacs and Pubmed databases, during the period between February and March 2010 ( $\mathrm{n}=12)$ (cont.)

\begin{tabular}{|c|c|c|c|c|c|c|c|c|c|c|c|}
\hline Drugs / ATC Code & 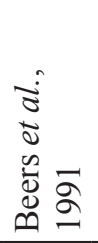 & $\begin{array}{l}\hat{\alpha} \\
\hat{\sigma} \\
\bar{d} \\
\bar{d} \\
\bar{n}\end{array}$ & 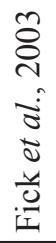 & 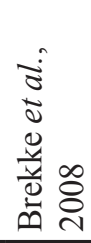 & 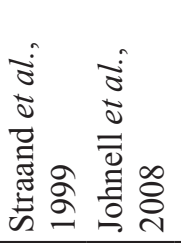 & 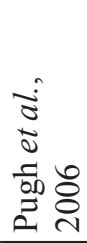 & 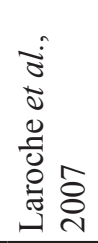 & 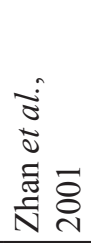 & 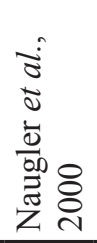 & 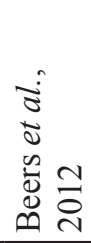 & 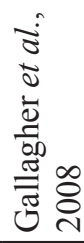 \\
\hline Castor oil (A06AB05) & & & $\mathrm{X}$ & & & & $\mathrm{X}$ & & & & \\
\hline Ranitidine (A02BA02) & $\mathrm{X}$ & & & & & & & & & & \\
\hline Muscle relaxants & & & & & & & & & & & $\mathrm{X}$ \\
\hline Baclofen (M03BX01) & & & & & & & $\mathrm{X}$ & & & & \\
\hline Carisoprodol (M03BA02) & $\mathrm{X}$ & $\mathrm{X}$ & $\mathrm{X}$ & $\mathrm{X}$ & & $\mathrm{X}$ & & $\mathrm{X}$ & & $\mathrm{X}$ & \\
\hline Cyclobenzaprine (M03BX08) & $\mathrm{X}$ & $\mathrm{X}$ & $\mathrm{X}$ & & & $\mathrm{X}$ & & $\mathrm{X}$ & & $\mathrm{X}$ & \\
\hline Chlorzoxazone (M03BB03) & & $\mathrm{X}$ & $\mathrm{X}$ & & & $\mathrm{X}$ & & $\mathrm{X}$ & & $\mathrm{X}$ & \\
\hline Metaxalone (none) & & $\mathrm{X}$ & $\mathrm{X}$ & & & $\mathrm{X}$ & & $\mathrm{X}$ & & $\mathrm{X}$ & \\
\hline Methocarbamol (M03BA03) & $\mathrm{X}$ & $\mathrm{X}$ & $\mathrm{X}$ & & & $\mathrm{X}$ & $\mathrm{X}$ & $\mathrm{X}$ & & $\mathrm{X}$ & \\
\hline Orphenadrine (M03BC01) & $\mathrm{X}$ & & $\mathrm{X}$ & & & $\mathrm{X}$ & & & & $\mathrm{X}$ & \\
\hline Solifenacin (G04BD08) & & & & & & & $\mathrm{X}$ & & & & \\
\hline Tolterodine (G04BD07) & & & & & & & $\mathrm{X}$ & & & & \\
\hline Ferrous sulphate (B03AA07) & $\mathrm{X}$ & $\mathrm{X}$ & $\mathrm{X}$ & & & & & & & & \\
\hline Theophylline (R03DA04) & & & & $\mathrm{X}$ & & & & & & & \\
\hline \multicolumn{12}{|l|}{ Treatments for dementia } \\
\hline Cyclandelate (C04AX01) & $\mathrm{X}$ & $\mathrm{X}$ & $\mathrm{X}$ & & & $\mathrm{X}$ & & & & & \\
\hline Isoxsuprine (C04AA01) & $\mathrm{X}$ & & $\mathrm{X}$ & & & $\mathrm{X}$ & & & & $\mathrm{X}$ & \\
\hline
\end{tabular}

TABLE II - Frequency of drugs considered potentially inappropriate medications (PIM) for elderly people, according to the instruments identified in Paho, Lilacs and Pubmed databases in February and March 2010, whose use should be avoided irrespective of the diagnosis or conditions of the patient

\begin{tabular}{lc}
\hline Instruments & PIM - N (\%) \\
\hline Beers (2012) & $118(72.4)$ \\
Fick DM, et al. (2003) & $73(44.7)$ \\
Laroche ML, et al. (2007) & $61(37.4)$ \\
Beers MH, et al. (1997) & $49(30.0)$ \\
Pugh MJV, et al. (2006) & $44(26.9)$ \\
Beers MH. (1991) & $38(23.3)$ \\
Zhan C, et al. $(2001)$ & $33(20.2)$ \\
Breeke M, et al. (2008) & $19(11.6)$ \\
Johnell K, et al. $(2008)$ & $10(6.1)$ \\
Straand J, et al. (1999) & $9(5.5)$ \\
Naugler CT, et al. (2000) & $4(2.4)$ \\
\hline Total of PIM & $163(100.0 \%)$ \\
\hline
\end{tabular}

ranitidine, spironolactone, estrogens, diazepam, digoxin, phenobarbital, haloperidol, hydrochlorothiazide, ibuprofen, insulin, dexchlorpheniramine, nifedipine, nitrofurantoin, doxazosin, methyldopa, mineral oil, ferrous sulphate, trihexyphenidyl). In this update, the only PIM that was removed from the list was testosterone. However, testosterone was maintained in the $17^{\text {th }}$ WHO Model List of Essential Medicines.

Moreover, among the 16 criteria identified, only eleven listed the drugs that should be avoided on the basis of diagnosis or the conditions of patient (drug interaction with the disease), the co-prescriptions that should be avoided in senescence and drugs for which is necessary to pay attention to the dose, duration and frequency of the treatment (Table III).

\section{DISCUSSION}

The pharmacotherapy prescribed, especially for the elderly population, should be effective and avoid potentially harmful drugs, since most of the adverse drug events appear in the post-commercialization period. Topinková et al. (2012) showed that strategies have been developed to target these goals which are focused on drug 
TABLE III - Potentially inappropriate medications (PIM) for elderly people listed by the instruments identified in Paho, Lilacs and Pubmed databases, during Februrary and March 2010, which are present in the Brazilian list of essential medicines (RENAME 2010) and in the $16^{\text {th }}$ WHO Model List of Essential Drugs, and their possible equivalent therapies.

\begin{tabular}{|c|c|c|c|c|c|}
\hline \multicolumn{3}{|c|}{ RENAME 2010} & \multicolumn{3}{|c|}{ WHO MODEL LIST } \\
\hline PIM & $\begin{array}{l}\text { Therapeutic } \\
\text { equivalent proposed }\end{array}$ & $\begin{array}{l}\text { The therapeutic } \\
\text { equivalent is listed } \\
\text { in Rename } 2010 ?\end{array}$ & PIM & $\begin{array}{l}\text { Therapeutic } \\
\text { equivalent }\end{array}$ & $\begin{array}{l}\text { The therapeutic } \\
\text { equivalent is in } \\
\text { WHO model list? }\end{array}$ \\
\hline amiodarone & verapamil & yes & amidarone & verapamil & yes \\
\hline amitriptyline & sertraline & no & amitriptilyne & sertraline & no \\
\hline clomipramine & sertraline & no & clorfenidramina & loratadine & no \\
\hline clonazepam & $\begin{array}{l}* \text { short-acting } \\
\text { benzodiazepine }\end{array}$ & no & clomipramine & sertraline & no \\
\hline chlorpromazine & quetiapine & yes & chlorpromazine & quetiapine & no \\
\hline diazepam & $\begin{array}{l}* \text { short-acting } \\
\text { benzodiazepine }\end{array}$ & no & diazepam & $\begin{array}{c}* \text { short-acting } \\
\text { benzodiazepine }\end{array}$ & yes \\
\hline digoxin & - & - & digoxin & - & - \\
\hline dexclorfenidramine & loratadine & yes & fluoxetine & sertraline & no \\
\hline estrogen & - & - & haloperidol & & - \\
\hline fluoxetine & sertraline & no & hydrochlorothiazide & - & - \\
\hline haloperidol & - & - & lorazepam & - & \\
\hline hydrochlorothiazide & - & - & methyldopa & losartan & no \\
\hline methyldopa & losartan & yes & nifedipine & - & yes \\
\hline $\begin{array}{l}\text { ciprionate } \\
\text { testosterone }\end{array}$ & - & - & nitrofurantoin & ciprofloxacin & yes \\
\hline nifedipine & - & yes & promethazine & loratadine & no \\
\hline nitrofurantoin & ciprofloxacin & yes & propranolol & - & - \\
\hline promethazine & loratadine & yes & ranitidine & omeprazole & yes \\
\hline propranolol & atenolol & yes & ferrous sulphate & - & - \\
\hline ranitidine & omeprazole & yes & testosterone & - & - \\
\hline ferrous sulphate & - & - & & & \\
\hline
\end{tabular}

*The dose in the elderly should be half or less than half the dose recommended for a young adult $<27>$.

appropriateness, adherence and adverse drug events. The authors highlight the medication review as one of the more promising interventions to assess the risk/benefit of geriatric pharmacotherapy. To promote this kind of safety assessment, our study identified the instruments that may be applied to assess the quality of medical prescription for elderly people.

However, since the criteria were developed in North America (Beers criteria and their updates) and Europe, there is a need to adapt an instrument of PIM classification to Brazilian conditions (Ribeiro et al., 2005), since $23 \%$ (38/165) of inappropriate geriatric medicines are not marketed in Brazil (ethacrynic acid, alimemazine, amobarbital, amoxapine, butalbital, carbutamide, cyamemazine, cyclandelate, clidinium-chlordiazepoxide, chlorprothixen, diflunisal, eszopiclone, guanabenz, guanadrel, guanfacine, guanethidine, halazepam, loflazepate, meprobamate, mesoridazine, metaxalone, methocarbamol, nicardipine, nordazepam, oxaprozin, pentazocine, perphenazine, prazepam, procainamide, prochlorperazine, propantheline, quazepam, secobarbital, temazepam, tetrazepam, tolmetin, trimethobenzamide, trimipramine). A review carried out by Corsonello et al. (2012) corroborates this argument. The authors noted that the START/STOPP criteria were developed to promote the prevention of inappropriate prescribing in European nations, since the 2003 updated Beers criteria include drugs that are not available in Europe or hardly ever are prescribed. Therefore, with the aid of Brazilian criteria, the prevalence of appropriate pharmacotherapy may be 
TABLE IV - List of potentially inappropriate medications (PIM) for the elderly that should be avoided as indicated by the clinical diagnosis of the patient (drug interaction with disease), the dose, frequency and duration of the drug administration and drug association, according to the methods identified in PAHO (available at: http://regional.bvsalud.org/php/index.php), Lilacs and Pubmed databases, during the period between February and March $2010(\mathrm{n}=10)$

\begin{tabular}{|c|c|c|c|c|c|c|c|c|c|c|}
\hline Drugs & 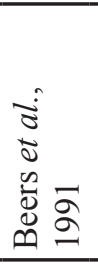 & 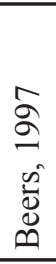 & 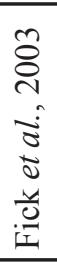 & 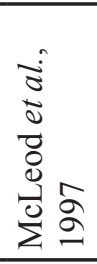 & 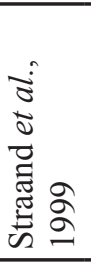 & 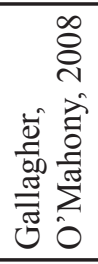 & 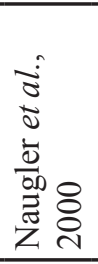 & 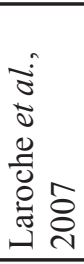 & 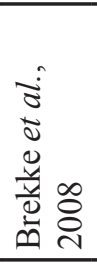 & 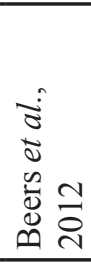 \\
\hline \multicolumn{11}{|l|}{$\overline{\text { Combinations }}$} \\
\hline NSAIDs + Diuretics & & & & & & & & & $\mathrm{X}$ & \\
\hline NSAIDS + Meperidine ou Pentazocine & & & & $\mathrm{X}$ & & & & & & \\
\hline NSAIDS + Piroxicam, Ketorola or Mefenamic acid & & & & $\mathrm{X}$ & & & & & & \\
\hline NSAIDS + ACEi & & & & & & & & & $\mathrm{X}$ & \\
\hline NSAIDS + SSRI & & & & & & & & & $\mathrm{X}$ & \\
\hline NSAIDS + Warfarin & & & & $\mathrm{X}$ & & $\mathrm{X}$ & & & $\mathrm{X}$ & \\
\hline Amitriptyline + Chlordiazepoxide & & $\mathrm{X}$ & $\mathrm{X}$ & & & & & & & $\mathbf{X}$ \\
\hline Amitriptyline + Perphenazine & $\mathbf{X}$ & $\mathbf{X}$ & $\mathbf{X}$ & & & & & & & $\mathbf{X}$ \\
\hline Anticholinergic + Antipsychotics & & & & $\mathrm{X}$ & & & $\mathrm{X}$ & & & \\
\hline Cimetidine + Warfarin & & & & $\mathrm{X}$ & & & & & & \\
\hline Clidinium + Chlordiazepoxide & & & $\mathrm{X}$ & & & & & & & $\mathbf{X}$ \\
\hline Corazepato + Acepromazine & & & & & & & & $\mathrm{X}$ & & \\
\hline Desclorfeniramina + Betamethasone & & & & & & & & $\mathrm{X}$ & & \\
\hline $\mathrm{SSRI}+\mathrm{MAOI}$ & & & & $\mathrm{X}$ & & & & & & \\
\hline Methyldopa + Hydrochlorothiazide & & $\mathrm{X}$ & $\mathrm{X}$ & & & & & & & \\
\hline \multicolumn{11}{|l|}{ Dose limits, frequency and duration of treatment } \\
\hline Acetylsalicylic acid $\geq 150 \mathrm{mg} /$ dia (dose) & & & & & & $\mathrm{X}$ & & & & \\
\hline Pain relievers containing codeine & & & & & $\mathrm{X}$ & & & & & \\
\hline Antibiotics prescribed for the first crisis of bronchitis & & & & & $\mathrm{X}$ & & & & & \\
\hline Gastrointestinal antispasmodics & & & & & $\mathrm{X}$ & & & & & \\
\hline 1st generation antihistamines (duration) & & & & & & $\mathrm{X}$ & & & & \\
\hline Antipsychotic drugs (dose, duration) & $\mathbf{X}$ & & & & $\mathrm{X}$ & & & & & \\
\hline Antipsychotics for nonpsychotic diagnoses & & & & & $\mathrm{X}$ & & & & & \\
\hline Barbiturates (duration) & & & & $\mathrm{X}$ & & & & & & \\
\hline Benzodiazepines (dose, duration) & $\mathbf{X}$ & & & $\mathrm{X}$ & $\mathrm{X}$ & $\mathrm{X}$ & & & & \\
\hline $\mathrm{H} 2$ blockers (dose. frequency and duration) & $\mathbf{X}$ & & & & & & & & & \\
\hline Decongestants (duration) & $\mathbf{X}$ & & & & & & & & & \\
\hline Digoxin (dose) & $\mathbf{X}$ & & & & & & & & & \\
\hline Iron supplements (dose and frequency) & $\mathbf{X}$ & & & & & & & & & \\
\hline Thiazides (dose) & $\mathbf{X}$ & & & & $\mathrm{X}$ & & & & & \\
\hline Three or more psychotropic drugs (duration) & & & & & & & & & $\mathrm{X}$ & \\
\hline \multicolumn{11}{|l|}{ Interaction Pathology } \\
\hline $\begin{array}{l}\text { Adenoma of the prostate, glaucoma and urinary retention } X \\
\text { anticholinergic }\end{array}$ & & & & & & & & $\mathrm{X}$ & & \\
\hline $\begin{array}{l}\text { Angina in patients with a history of asthma, COPD or heart } \\
\text { failure X } \beta \text {-blockers }\end{array}$ & & & & $\mathrm{X}$ & & & & & & \\
\hline Anorexia and malnutrition $\mathrm{X}$ central nervous system stimulants & & & $\mathrm{X}$ & & & & & & & \\
\hline Conductive cardiac abnormalities $\mathrm{X}$ tricyclic antidepressant & & & & & & $\mathrm{X}$ & & & & \\
\hline Arrhythmias X tricyclic antidepressants & & & $\mathrm{X}$ & & & & & & & \\
\hline $\begin{array}{l}\text { No history of coronary symptoms, cerebral or peripheral vascular } \\
\text { occlusive or event X Acetylsalicylic acid }\end{array}$ & & & & & & $\mathrm{X}$ & & & & \\
\hline
\end{tabular}


TABLE IV - List of potentially inappropriate medications (PIM) for the elderly that should be avoided as indicated by the clinical diagnosis of the patient (drug interaction with disease), the dose, frequency and duration of the drug administration and drug association, according to the methods identified in PAHO (available at: http://regional.bvsalud.org/php/index.php), Lilacs and Pubmed databases, during the period between February and March 2010 (n=9) (cont.)

\begin{tabular}{|c|c|c|c|c|c|c|c|c|c|c|c|c|c|c|c|}
\hline Drugs & 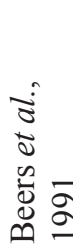 & $\begin{array}{l}\hat{\sigma} \\
\hat{\sigma} \\
\hat{\infty} \\
\dot{0} \\
\infty\end{array}$ & 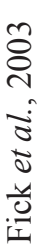 & 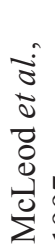 & ลू & 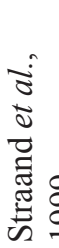 & & 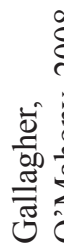 & 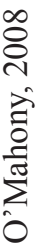 & 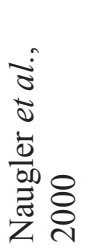 & 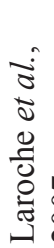 & 응 & 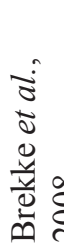 & @ & 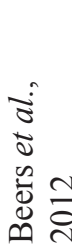 \\
\hline
\end{tabular}

Chronic constipation without concomitant use of laxatives X opioids for more than 2 weeks

Constipation $\mathrm{X}$ tricyclic antidepressant

Seizures X clozapine, chlorpromazine, thioridazine and thiothixene

$\mathrm{X}$

$\mathrm{X}$

X

Dementia $\mathrm{X}$ anticholinergics, trihexafenidil, tropatepina, biperiden, neuroleptics (except risperidone and olanzapine) and benzodiazepines

Depression $\mathrm{X}$ active metabolites with tricyclic antidepressant (eg amitriptyline or imipramine)

Depression X long-acting benzodiazepines and sympatholytic agents

Depression X methylphenidate

Clotting disorders X NSAIDs, dipyridamole, ticlopidine and clopidogrel

Diabetes mellitus and frequent hypoglycemia X $\beta$-blockers

Diabetes X corticoids

Diarrhea or gastroenteritis of unknown cause severe $\mathrm{X}$ diphenoxylate, loperamide or codeine

Parkinson's Disease X Metoclopramide, conventional antipsychotics, tacrine

Raynaud's DiseaseX prolonged use of $\beta$-adrenergic blocker

Moderate pain X long-term use of potent opioids as a first treatment option

COPD X beta blockers and benzodiazepines long-term

COPD X theophylline

Muscle spasms X cyclobenzaprine or methocarbamol

Stress $\mathrm{X}$ benzodiazepínios beta-blockers and long acting

Atrial fibrillation $\mathrm{X}$ disopyramide

Drop $\mathrm{X}$ thiazide diuretic

Drop X indomethacin

Benign prostatic hyperplasia, glaucoma or cardiac arrest $\mathrm{X}$ tricyclic antidepressant Moderate to severe hypertension X NSAIDS

Hypertension X phenylpropanolamine, pseudoephedrine and amphetamines

Hypertension X reserpine

Postural hypotension X tricyclic antidepressants

Postural hypotension $\mathrm{X}$ chlorpromazine

Urinary incontinence $\mathrm{X}$ urapidil and prazosin

Insomnia X decongestants, theophylline, methylphenidate, MAOI, amphetamine
$\mathrm{X}$

$\mathrm{X}$

X

X $\mathrm{X}$

X

$\mathrm{X}$

$\mathrm{X}$

X $\quad X \quad X$

$\mathrm{X}$

$\mathrm{X}$

$\mathrm{X}$

$\begin{array}{lll}X & X & X \\ & X & x\end{array}$

X

$\mathrm{X}$

$\mathrm{X}$

$\begin{array}{lll}X & X & X\end{array}$

$\mathrm{X}$

$\mathrm{X}$

$\mathrm{X}$

$\mathrm{X} \quad \mathrm{X} \quad \mathrm{X}$

$\mathrm{X}$

$\mathrm{X}$

$\mathrm{X}$

$\mathrm{X}$

$\mathrm{X}$ 
TABLE IV - List of potentially inappropriate medications (PIM) for the elderly that should be avoided as indicated by the clinical diagnosis of the patient (drug interaction with disease), the dose, frequency and duration of the drug administration and drug association, according to the methods identified in PAHO (available at: http://regional.bvsalud.org/php/index.php), Lilacs and Pubmed databases, during the period between February and March 2010 (n=9) (cont.)

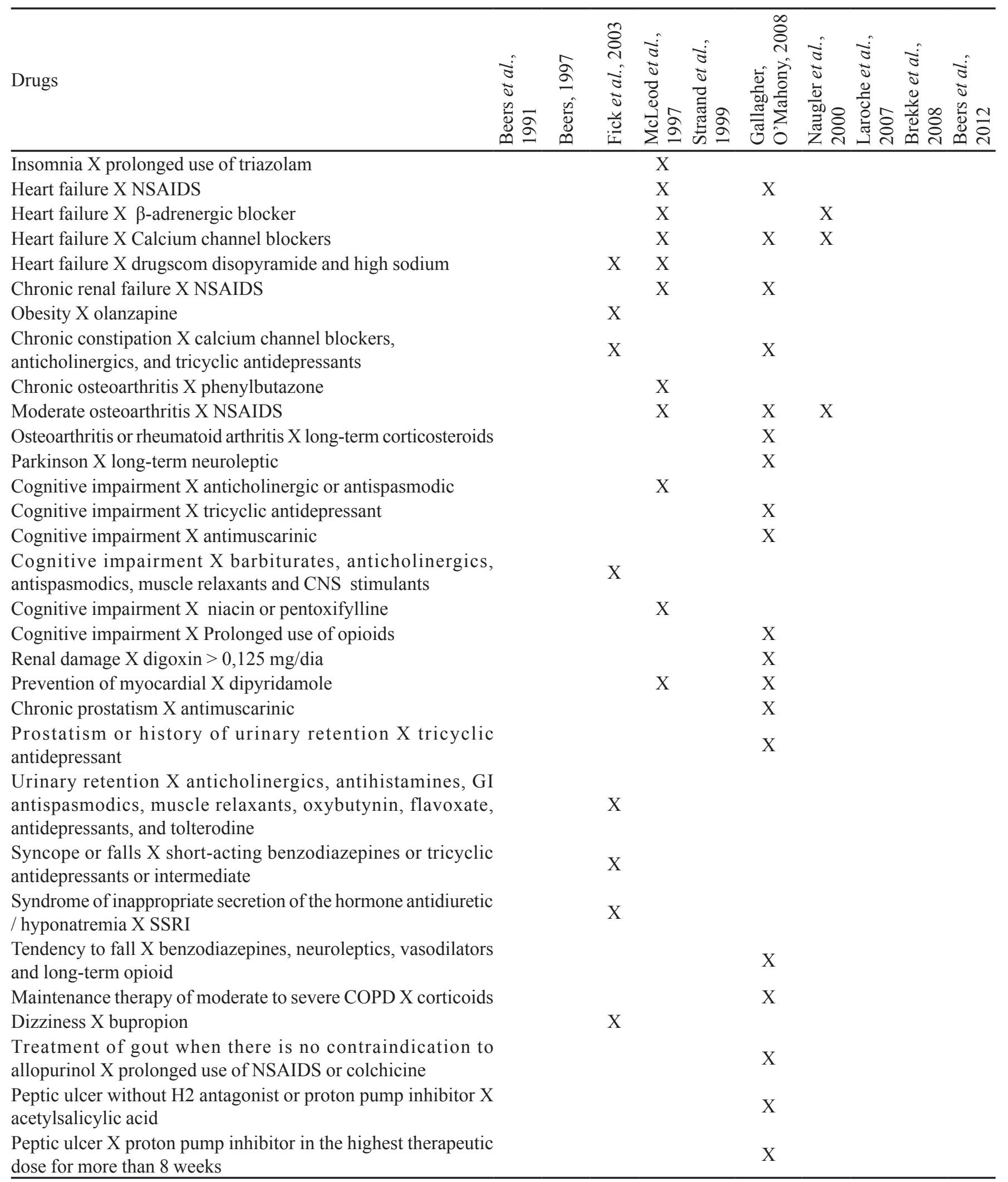


TABLE IV - List of potentially inappropriate medications (PIM) for the elderly that should be avoided as indicated by the clinical diagnosis of the patient (drug interaction with disease), the dose, frequency and duration of the drug administration and drug association, according to the methods identified in PAHO (available at: http://regional.bvsalud.org/php/index.php), Lilacs and Pubmed databases, during the period between February and March 2010 (n=9) (cont.)

\begin{tabular}{|c|c|c|c|c|c|c|c|c|c|c|}
\hline Drugs & 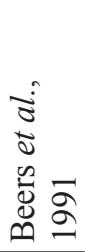 & 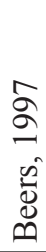 & 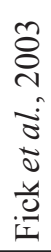 & 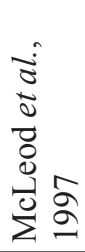 & 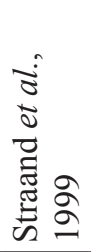 & 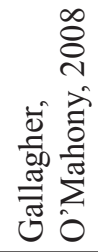 & 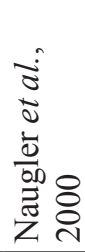 & 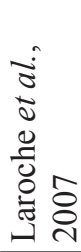 & 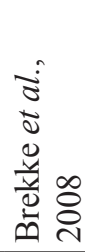 & 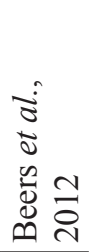 \\
\hline$\overline{\text { UlcerX NSAIDS }}$ & & & $\mathrm{X}$ & $\mathrm{X}$ & & $\mathrm{X}$ & $\mathrm{X}$ & & & \\
\hline \multicolumn{11}{|l|}{ Questionable efficacy } \\
\hline Benzodiazepines & & & & & & & $\mathrm{X}$ & & & \\
\hline $\begin{array}{l}\text { Drugs that act in the gastrointestinal tract with anticholinergic } \\
\text { properties }\end{array}$ & & & & & & & $\mathrm{X}$ & & & \\
\hline Cerebral vasodilator & & & & & & & $\mathrm{X}$ & & & \\
\hline
\end{tabular}

NSAIDs: nonsteroidal anti-inflammatory drugs; COPD: chronic obstructive pulmonary disease; ACEI: angiotensin-convertingenzyme inhibitor; MAOI: monoamino oxidase inhibitor; SSRI: selective serotonin reuptake inhibitor; CNS: central nervous system.

robustly assessed, allowing interventions to decrease medication prescribing errors.

Furthermore, the inclusion of safer equivalent therapies for the elderly in the list of Brazilian essential medicines may be another approach able to promote the safe use of drugs for the elderly, as well as to increase their access to less harmful medicines. This measure could have a great impact in developing countries, where most of the population is attended in public health care services, which commonly use RENAME to standardize their drugs. In Brazil, the total coverage of the public health service is $70 \%$ (IBGE, 2009) and approximately $20 \%$ of PIM are essential medicines (RENAME 2012). Although they meet the criteria of quality, safety, effectiveness and low cost for the majority of population, they are considered unsafe for elderly people. Therefore, the RENAME should make an assessment of the cost/benefit of the inclusion of safer alternatives for this age group, presented as a safety indicator of Pharmaceutical Assistance Management.

It is important to mention that there is no safer equivalent therapies for seven of the PIM considered essential medicines. In these cases, the development of clinical protocols clarifying the conditions of use and the measures required to avoid harming the health is essential. In addition pharmacotherapeutic follow-up may optimize the treatment, since it allows early recognition of negative clinical outcomes and may avoid therapeutic failure by noncompliance (Maust et al., 2013).

Finally, particular care should be taken with PIM that are over the counter (OTC) drugs (salicylic acid, ibuprofen, naproxen, belladonna alkaloids, scopolamine, dexchlorpheniramine, doxylamine, bisacodyl, cáscara sagrada, mineral oil, castor oil, ferrous sulphate, brompheniramine, carbinoxamine, cyproheptadine, chlorpheniramine, phenylephrine, carisoprodol, orphenadrine). This fact highlights the role of pharmacists in providing quality information regarding the correct use of drugs. Recently, the Brazilian Pharmacy Federal Council approved the pharmaceutical prescribing of OTC drugs (CFF, 2013), so that the treatment of minor disorders in drugstores is feasible. In this setting, the pharmacist should consider the risk/benefit of using a drug, the clinical conditions of the patient (renal or hepatic impairments, allergies, morbidities and comorbidities that contraindicate its use) and the safety of the drug to choose the best option for the user. Therefore, it is important aware them regarding the sanitary risk involving the use of PIM by elderly people.

\section{Study limitations}

The data may be underestimated because of selection bias, due to: 1) the search strategy in the databases; 2) data collection period; 3 ) restriction os search to; 4) articles selected for analysis being written in Spanish, English and Portuguese languages alone, and 5) 26 [12.8\% (26/203)] manuscripts being unavailable for analysis. Therefore, some other criteria developed to assess the quality of drugs prescribed for elderly people may not have been identified by the methods used in this study.

\section{CONCLUSION}

The Brazilian register of essential medicines (RENAME) lists effective, safe and economic drugs. 
However, approximately $25 \%$ of them are considered potentially inappropriate for elderly people. Therefore, they do not meet the safety attribute for this age group, most of whom are attended in public health care services and, in general, use polypharmacy. Thus, it is necessary for less noxious drugs for this population to be included in RENAME, in order to provide access to more effective treatments with less harmful potential. In this setting, the pharmacist can contribute to the safety assessment and follow-up of geriatric pharmacotherapy, mainly for those PIM that have no safer equivalent therapies (digoxin, estrogen, haloperidol, hydrochlorthiazide, ciprionate testosterone, nifedipine and ferrous sulphate), as well as for those that are OTC drugs whose use is not exempt from counseling. These measures would meet the national policy of risk management. Furthermore, the intensive monitoring of medicines allows the establishment of safety indicators for Pharmaceutical Assistance.

\section{REFERENCES}

AMERICAN Geriatrics Society. American Geriatrics Society updated Beers Criteria for potentially inappropriate medication use in older adults. J. Am. Geriatr. Soc., v.60, n.4, p.616-631, 2012.

BARRY, P.J.; GALLAGHER, P.; O’MAHONY, D. START (screening tool to alert doctors to the right treatment) an evidence based screening tool to detect prescribing omissions in elderly patients. Age Ageing, v.36, n.6, p.632$638,2007$.

BEERS, M.H. Explicit criteria for determining pottencially inappropriate medication use by the elderly: an update. Arch Intern. Med., v.157, n.14, p.1531-1536, 1997.

BEERS, M.H.; OUSLANDER, J.G.; ROLLINGHER, J.; REUBEN, D.B.; BECK, J.C. Explicit criteria for determining inappropriate medication use in nursing home residents. Arch Intern. Med., v.151, n.9, p.1825-1832, 1991.

BONGUE, B.; NAUDIN, F.; LAROCHE, M.L.; GALTEAU, M.M.; GUY, C.; GUÉGUEN, R. Trends of the potentially inappropriate medication consumption over 10 years in older adults in east of France. Pharmacoepidemiol. Drug Saf., v.18, n.12, p.1125-1133, 2009.

BRASIL. IBGE. Projeção da população por sexo e grupos de idade, em $1^{\circ}$ de julho - 2000-2060. Available at: <http:// www.ibge.gov.br/home/estatistica/populacao/projecao_da populacao/2013/default.shtm $>$. Accessed on: 29 Sept. 2013.
INSTITUTO brasileiro de geografia e estatística. Indicadores sociodemográficos e de saúde. Rio de Janeiro: Ministério do Planejamento, Orçamento e Gestão, 2009. 152 p. Available at: <http://www.ibge.gov.br/home/estatistica/populacao/ indic_sociosaude/2009/indicsaude.pdf $>$. Accessed on: 18 Oct. 2013.

BRASIL. Leis, etc. Lei n. 8.842 de 4 de janeiro de 1994. Dispõe sobre a Política Nacional do Idoso, cria o Conselho Nacional do Idoso e dá outras providências. Diário Oficial da União, Brasília, 5 jan.1994. Seção 1. Available at: $<$ http:// www.planalto.gov.br/ccivil_03/leis/18842.htm $>$. Accessed on: 18 Oct. 2013.

BRASIL. Ministério da Saúde. Secretaria de Ciência, Tecnologia e Insumos Estratégicos. Departamento de Assistência Farmacêutica e Insumos Estratégicos. Relação nacional de medicamentos essenciais. 7.ed. Brasilia, 2010. Available at: <http://portal.saude.gov.br/portal/arquivos/ pdf/rename2010final.pdf $>$. Accessed on: 24 Sept. 2013.

BRASIL. Senado Federal. Comissão Diretora. Parecer n.1301 de 2003. Estatuto do Idoso. Projeto de Lei da Câmara n.57. Brasília, 2003. 28 p. Available at: <https://www.nescon. medicina.ufmg.br/biblioteca/imagem/2051.pdf $>$. Accessed on: 18 Oct. 2013.

BRASIL. Ministério da Saúde. Secretaria de Ciência, Tecnologia e Insumos Estratégicos. Departamento de Assistência Farmacêutica e Insumos Estratégicos. Relação nacional de medicamentos essenciais. 8.ed. Brasilia, 2012. Available at: <http://portal.saude.gov.br/portal/arquivos/ pdf/CONITECANEXOSRENAME.pdf $>$. Accessed on: 24 Sept. 2013.

BREKKE, M.; ROGNSTAD, S.; STRANND, J.; FURU, K.; GJELSTAD, S.; BJORNER, T. Pharmacologically inappropriate prescriptions for elderly patients in general practice: How commom? Baseline data from the prescription peer academic detailing (Rx-PAD) study. Scand. J. Prim. Health Care, v.26, n.2, p.80-85, 2008.

CASTELINO, R.L.; BAJOREK, B.V.; CHEN, T.F. Targeting suboptimal prescribing in the elderly: a review of the impact of pharmacy services. Ann. Pharmacother, v.43, n.6, p.1096-1106, 2009.

CONSELHO Federal de Farmácia. Regula a prescrição farmacêutica e dá outras providências. Resolução ${ }^{\circ} 586$, de 29 de agosto de 2013. Diário Oficial da União. Brasília, 26 de setembro de 2013. 
CORSONELLO, A.; PEDONE, C.; INCALZI, A. Age-related pharmacokinetic and pharmacodynamic changes and related risks of adverse drug reactions. Curr. Med. Chem., v.17, n.6, p.571-584, 2010.

CORSONELLO, A.; ONDER, G.; ABBATECOLA, A.M.; GUFFANTI, E.E.; GARERI, P.; LATTANZIO, F. Explicit criteria for potentially inappropriate medications to reduce the risk of adverse drug reactions in elderly people: from Beers to STOPP/START criteria. Drug Saf., v.35, n.1, p.21-28, 2012.

DE PAULA, T.C.; BOCHNER, R.; MONTILLA, D.E.R. Clinical and epidemiological analysis of hospitalizations of elderly due to poisoning and adverse effects of medications, Brazil from 2004 to 2008. Rev. Bras. Epidemiol., v.15, n.4, p.828-844, 2012.

FIALOVÁ, D.; ONDER, G. Medication errors in elderly people: contributing factors and future perspectives. Br. J. Clin. Pharmacol., v.67, n.6, p.641-645, 2009.

FICK, D.M.; COOPER, J.W.; WADE, W.E.; WALLER, J.L.; MACLEAN, J.R.; BEERS, M.H. Updating the beers criteria for potentially inappropriate medication use in older adults: results of a US consensus panel of experts. Arch. Intern. Med., v.163, n.22, p.2716-2722, 2003.

GALLAGHER, P.; O’MAHONY, D. STOPP (Screening Tool of Older Persons' Potentially Inappropriate Prescriptions): application to acutely ill elderly patients and comparison with Beers' criteria. Age Ageing, v.37, n.6, p.673-679, 2008

GALLAGHER, P.; RYAN, C.; BYRNE, S.; KENNEDY, J.; O'MAHONY, D. STOPP (Screening Tool of Older Person's Prescriptions) and START (Screening Tool to Alert doctors to Right Treatment). Consensus validation. Int. J. Clin. Pharmacol. Ther., v.46, n.2, p.72-83, 2008.

GORDILHO, A.; SÉRGIO, J.; SILVESTRE, J.; RAMOS, L.R.; FREIRE, M.P.A.; ESPINDOLA, N. Desafios a serem enfrentados no terceiro milênio pelo setor saúde na atenção integral do idoso. Rio de Janeiro: Universidade do Estado do Rio de Janeiro, Universidade Aberta da Terceira Idade, 2000. 86p. Available at: $<$ http://www. observatorionacionaldoidoso.fiocruz.br/biblioteca/_ manual/15.pdf $>$. Accessed on: 18 Oct. 2013.

HANLON, J.T.; SCHMADER, K.E.; SAMSA, G.P.; WEINBERGER, M.; UTTECH, K.M.; LEWIS, I.K. A method for assessing drug therapy appropriateness. J. Clin. Epidemiol., v.45, n.10, p.1045-1051, 1992.
JOHNELL, K.; FASTBOM, J. Multi-dose drug dispensing and inappropriate drug use: a nationwide register-based study of over 70000 elderly. Scand. J. Prim. Health Care, v.26, n.2, p.86-91, 2008.

LAROCHE, M.L.; CHARMES, J.P.; MERLE, L. Potentially inappropriate medication in the elderly: a French consensus panel list. Eur. J. Clin. Pharmacol., v.63, n.8, p.725-731, 2007.

LIPTON, H.L.; BIRD, J.A.; BERO, L.A.; MCPHEE, S.J. Assessing the appropriateness of physician prescribing for geriatric outpatients: development and testing of an instrument. J. Pharm. Technol., v.9, n.3, p.107-113, 1993.

MAUST, D.T.; MAVANDADI. S.; BENSON, A.; STREIM, J.E.; DIFILIPPO, S.; SNEDDEN. T.; WEBER, A.L.; OSLIN, D.W. Telephone-based care management for older adults initiated on psychotropic medication. Int. J. Geriatr. Psychiatry, v.28, n.4, p.410-416, 2013.

MCLEOD, P.J.; HUANG, A.R.; TAMBLYN, R.M.; GAYTON, D.C. Defining inappropriate practices in prescribing for elderly people: national consensus panel. CMAJ, v.156, n.3, p.385-391, 1997.

NAUGLER, C.T.; BRYMER, C.; STOLEE, P.; ARCESE, Z.A. Development and validation of an improving prescribing in the elderly tool. Can. J. Clin. Pharmacol., v.7, n.2, p.103$107,2000$.

PUGH, M.J.V.; HANLON, J.T.; ZEBER, J.E.; BIERMAN, A.; CORNELL, J.; BERLOVITZ, D.R. Assessing potentially inappropriate prescribing in the elderly veterans affairs population using the HEDIS 2006 quality measure. $J$. Manag. Care Pharm., v.12, n.7, p.537-545, 2006.

RIBEIRO, A.Q.; ARAÚJO, C.M.C.; ACURCIO, F.A.; MAGALHÃES, S.M.; CHAIMOWICZ, F. Quality assessment of drug use in the elderly: a review of available evaluation methods. Ciênc. Saúde Coletiva, v.10, n.4, p.1037-1045, 2005.

SMET, P.A.; DENNEBOOM, W.; KRAMERS, C.; GROL, R. A composite screening tool for medication reviews of outpatients: general issues with specific examples. Drugs Aging, v.24, n.9, p.733-760. 2007.

SPINEWINE, A.; SCHMADER, K.E.; BARBER, N.; HUGHES, C.; LAPANE, K.L.; SWINE, C. Appropriate prescribing in elderly people: how well can it be measured and optimised? Lancet., v.370, n.13, p.173-184, 2007. 
STRAAND, J.; ROKSTAD, K.S. Elderly patients in general practice: diagnoses, drugs and inappropriate prescriptions. Fam. Pract., v.16, n.4, p.380-388, 1999.

TOPINKOVÁ, E.; BAEYENS, J.P.; MICHEL, J.P.; LANG, P.O. Evidence-based strategies for the optimization of pharmacotherapy in older people. Drugs Aging, v.29, n.6, p.477-494, 2012.

UNITED NATIONS SECRETARIAT. Department of Economic and Social Affairs. Population Division. Programme of action. In: International Conference on Population and Development. 1994. New York: ST/ ESA/SER, 1999. Available at: <http://www.un.org/en/ development/desa/population/events/conference/index. shtml>.Accessed on: 20 jan 2011.
WHO. Model list of essential medicines: 16 ${ }^{\mathrm{a}}$ list. 2010. Available at: <www.who.int/medicines/publications/ essentialmedicines/Updated_sixteenth_adult_list_en.pdf $>$. Accessed on: 20 Nov. 2010.

WORLD Health Organization. Perspectivas politicas de la OMS sobre medicamentos. La farmacovigilancia: garantía de seguridad en el uso de los medicamentos. Ginebra, 2004. p.6.

ZHAN, C.; SANGL, J.; BIERMAN, A.S.; MILLER, M.R.; FRIEDMAN, B.; WICKSER, S.W. Potentially inappropriate medication use in the community-dwelling elderly: findings from the 1996 medical expenditure panel survey. JAMA, v.286, n.22, p.2823-2829, 2001.

Received for publication on $23^{\text {rd }}$ March 2013 Accepted for publication on $12^{\text {th }}$ November 2013 